\title{
Reference ID
}

National Cancer Institute

\section{Source}

National Cancer Institute. Reference ID. NCI Thesaurus. Code C82531.

A sequence of characters that represents a reference identification. 